\title{
Costs and methods of preventive visual screening and the relation between esotropia and increasing hypermetropia
}

\author{
H.J. SIMONSZ, B. GROSKLAUSER, S. LEUPPI \\ Department of Strabismus \& Neuro-ophthalmology, Eye Clinic, Kantonsspital, Sankt-Gallen, \\ Switzerland and the Netherlands Ophthalmic Research Institute, Amsterdam, The Netherlands
}

Accepted 1 September 1992

Key words: Amblyopia, Baby-glasses, Early visual screening, Hypermetropia

\begin{abstract}
Atkinson has shown that early correction of hypermetropia reduces the incidence of esotropia. If esotropia is reduced by prescribing glasses early, the rate of esotropia-induced amblyopia can be similarly reduced; this would have important economic consequences. We have studied (1) how costs compare to benefits in early visual screening, (2) how videorefraction as used by Atkinson compares to retinoscopy, and (3) whether esotropia is more likely to occur in children who have increasing as opposed to decreasing hypermetropia. The costs of the study so far have been high. It was exceedingly difficult to get all infants invited, come to the clinic and examined. Videorefraction did not compare favourably with retinoscopy in terms of costs and precision, whereas the amount of skill and time needed was approximately equal. The third question, whether esotropia is more likely to occur in children who have increasing as opposed to decreasing hypermetropia, arose from the controversy whether, in the general population, refraction increases or decreases during the first years of life. We found that papers reporting a decrease of hypermetropia in early childhood were studies of large cross-sections of the general population, whereas papers that reported an initial increase originated from ophthalmological practices or strabismus departments. These conflicting results could be reconciled by assuming a population bias: if esotropia is more likely to occur in children with increasing hypermetropia, children with increasing hypermetropia will preferentially be seen by ophthalmologists. It seems natural that children with increasing hypermetropia are more likely to squint, because additional accommodation, needed to overcome increasing hypermetropia, will inevitably confer additional convergence. This relationship has meanwhile been confirmed by others.
\end{abstract}

\section{Introduction}

Dr. Atkinson of Cambridge University has demonstrated that early detection and correction of hypermetropia reduces the incidence of convergent strabismus [1]. She found 5\% hypermetropia (defined as $>4$ diopters) in 6to 9-month-old infants. In the study half of these hypermetropic children were given glasses. At age 4, $21 \%$ of the uncorrected hypermetropes had esotropia, against $6.2 \%$ of the corrected hypermetropes. In addition, slight bilateral amblyopia was noted at age 4 that was greater in uncorrected than in corrected hypermetropes. 
This bilateral, refractional amblyopia is not likely to have important consequences later in life. On the other hand, it is to be expected that, when the incidence of strabismus among hypermetropes is reduced to a third by prescribing glasses early, the rate of strabismus-induced amblyopia could be similarly reduced. This would have a large economic impact. The result of the study is of great importance, especially because Dr. Atkinson's study is the largest and best controlled of its kind ever. In the study, a "Videorefractor' is used to measure refraction in the infants. Two out-of-focus pictures of the child are taken with a video camera, its lens being set at 0.5 and 1.5 metres, in addition to an orienting in-focus picture, with the lens set at 0.75 meter. A dot-shaped flash is mounted coaxially on the camera lens. The sizes of the images of the blurred pupils vary depending on the refractive error of the eye (actually using the principle of retinoscopy 'in reverse').

At the second Meeting of the Child Vision Research Society in Cambridge in 1989, Dr. Atkinson made the suggestion that possibly up to half of the amblyopia cases and half of the strabismus cases could be prevented at a price of UK $£ 1$ per capita. Considering the costs of treatment of amblyopia and strabismus, the professional limitations of unilateral amblyopes and the costs incurred when unilateral amblyopes lose their better eye, further study of the applicability of early visual screening seemed therefore imperative. Hence, in our study described below, the first question we tried to answer is whether early visual screening can be carried out in a general population and at a reasonable price. A second question we studied is whether esotropia is more likely to occur in children who have increasing hypermetropia than in children who have decreasing hypermetropia.

\section{Methods}

The study was supported by a grant from the Swiss Union for Prevention of Blindness, enabling us to hire an orthoptist one day per week for the period of 2 years. In the Department of Obstetrics at the Kantonsspital Sankt Gallen, where almost all children from the city of Sankt Gallen are born, the parents of newborn children received an information sheet. When the children were 6 to 9 months of age, the parents were invited to come to our department for a free examination. In addition to videorefraction in cycloplegia, funduscopy was performed on all the children. In case of abnormalities, the family ophthalmologist was informed and advised to perform retinoscopy and prescribe baby-glasses. Retinoscopy could also be done at our department, if the ophthalmologist so wished, but these children were in the end always referred back to the ophthalmologist.

As it has in the mean time been found (see Discussion) that increasing hypermetropia is a more important risk factor for the development of amblyopia than hypermetropia per se, in our study we later determined 
refraction twice, with a six-month interval, in order to be able to estimate the need for glasses more precisely, and prescribe glasses to all children with increasing hypermetropia.

\section{Results}

Costs of the preparation of the study. The costs of the preparation-phase of the study have been very high. Apart from the salary of the part-time orthoptist, there have been many other costs, partly unexpected, unforeseen or underestimated.

Firstly, there was the problem of the baby-glasses. We understand that getting a pair of good and light baby-glasses is also a major problem in other countries. The baby-glasses that are available in Germany and Switzerland are extremely good but, because of very high manufacturing costs, so expensive that not much cooperation on the part of the parents was to be expected in this regard. We had extensive discussions with the manufacturer of these high quality baby-glasses and finally they were willing, because of the study, to offer the first two pairs of baby-glasses for each child under conditions normally applied to low-vision aids: the frame and the glasses were to be delivered ready ground and mounted by this manufacturer for a fixed, low price. On the one hand, this meant less work for the opticians but, on the other hand, guaranteed the opticians a satisfied customer, because the best and lightest combination of frame and glasses was used. The opticians agreed to this procedure. Almost all babies were fitted with excellent, light-weight baby-glasses, that were generally well accepted.

After that, we had extensive discussions with the ophthalmologists in our region. They turned out to be very sceptical. Had this study also been performed elsewhere? Why do we have to be the first, can't we wait until this is all standard procedure in other countries? Has the Swiss Ophthalmological Society been informed of this procedure? Isn't it dangerous to wear glasses at this age, what if the child harms itself with the glasses? Will these children wear these glasses? Is it really worth all the trouble?

Some nervousness among the ophthalmologists may also have arisen from the prospect of a waiting room full of infants awaiting retinoscopy. Alternatively, some ophthalmologists may have feared competition, although we explicitly expressed our intention to refer all children with abnormalities to the family ophthalmologist for retinoscopy and prescription of glasses. Peadiatricians examined some of the infants born, and these infants were more likely to be children with defects. So we informed them by means of a talk and information leaflets, asking them to inform the parents of the possibility of our free eye examination. There has been a moderate response. 
Next, we presented our plans to the opticians. They were very enthusiastic even though, as stated above, part of their work, in the delivery of the first two pairs of glasses, was being taken from them. The need for research in the field of preventive visual screening was well understood by opticians, much better than by ophthalmologists.

Then we had talks with the Department of Preventive Medicine of the Government of the Canton. They were very interested in the study and offered many helpful suggestions. The general 'Invalids' insurance, a very large institution in Switzerland, to whom we sent a copy of our first information mailing, was quick to respond that the current guidelines for reimbursement, i.e. a contribution to the cost of glasses if vision was below 0.2 or fixation was eccentric, were to be strictly adhered to.

The largest problem is how to get all the infants invited, come to the clinic, and examined. In the Canton Sankt Gallen 5300 children are born each year, 1500 of whom are born in the city of Sankt Gallen. How could we possibly reach all of them?

First, we talked extensively with the nurses of the Mothers' information offices. These offices are manned by nurses, not by doctors. They are very small, usually a single room for both waiting and examinations. Our suggestion was to visit each office, one afternoon every three months, and examine all infants aged 6 to 9 months who attended that particular office. This plan proved impossible, simply because of lack of space. In the end, the union in charge of these offices also turned down our second plan: to have the nurses inform the parents of the possibility of our free eye examination. Indeed, who is going to pay for the 5 extra minutes per child needed to inform the parents, at a rate of 5300 children per year?

The education councils were a second option. The preventive orthoptic examinations of \pm 5 -year-olds that have been carried out by our department for years are paid for by these councils but, unfortunately, they do not concern themselves with younger children.

A third possibility would be to ask all city councils to provide a room once every three months, and to invite all parents of 6- to 9-month-old infants to come there for free eye examination. This procedure is not expected to meet with much cooperation, partly due to the autonomous and decentralized government system in Switzerland. For instance, each city council would have to vote separately in a lengthy procedure.

The only remaining possibility was the one described in the Methods section: via the Department of Obstetrics, where most of the children of the city of Sankt Gallen are born. All this will certainly not lead to a $100 \%$ coverage. It was additionally sobering to hear from Dr. Todter (Sankt Pölten, Austria) that Austrian mothers, even though they get a financial reward if they have their children examined, often fail to show up for a second or third examination, especially if it concerns the third or later child. 


\section{Discussion}

Benefits. What are the benefits of early visual screening? First, it is important to realize that $\pm 2 \%$ of the population has amblyopia and $\pm 0.175 \%$ of these persons loses the better eye during his or her working career [2]. Loss of the only good eye in cases where treatment of amblyopia was delayed or otherwise unsuccessful is a rare event. On the other hand, the costs incurred in these cases because of the grave disability are extremely high. $34 \%$ of the children that are first referred for amblyopia are age 5 or older [3], and the prognosis of amblyopia is certainly worse at this age. It is possible that the incidence of amblyopia in the adult population can be reduced by tracing and treating amblyopia at an earlier age. Large savings could also be obtained by reducing the workload of orthoptists and strabologists: if, as Dr Atkinson found, two thirds of the strabismus and amblyopia in hypermetropes is prevented by prescribing glasses to hypermetropic infants, treatment of amblyopia and surgery can be totally avoided in these cases. Lack of binocularity per se probably has little economic impact: from an economic standpoint it is probably irrelevant whether certain professions, such as bus driving, are closed for a small minority of the population because of a visual handicap. The economic impact of a slightly increased chance of having a car accident, for instance, because of lack of binocular vision, is also probably small.

Videorefraction. Videorefraction did not compare favourably with retinoscopy in terms of costs and precision. Apart from being an expensive apparatus (Sfr. 23500), handling it needed considerable skill, and the question arose, how much the skill needed to handle the Videorefractor differs from the skill needed for retinoscopy. We felt that orthoptic training is necessary for reliable operation of the Videorefractor, as some familiarity with clinical optical devices, strabimus (angle kappa, in particular) and optics is required. It is probably cheaper to teach an orthoptist retinoscopy. Curiously, the cost-benefit analysis of early refractive screening then depends to a large extent on the relation between ophthalmologists, orthoptists and optometrists in each country. In England, Belgium and Switzerland, orthoptists do not perform retinoscopy, because either the number of ophthalmologists is sufficient to do all retinoscopies or, as in England, optometrists do some of them. In other countries with a shortage of ophthalmologists as in Holland, most retinoscopies in children are done by orthoptists.

Videorefraction took about 10 minutes per infant. We understand that 5 minutes should be possible. However, we are certain that most Dutch orthoptists can get a good impression of the refraction of both eyes of an infant in five minutes with retinoscopy, so we think the discussion is still open.

In newer versions of the videorefractor, like the Topcon version, some of 
the problems have been overcome, for example, with a device to elicit coaxial fixation, i.e. the infant looks straight into the lens, and image processing software to evaluate the image of the blurred pupil.

Esotropia is more likely to occur when hypermetropia increases. The third question was whether esotropia is more likely to occur in children who have increasing as opposed to decreasing hypermetropia. This question arose from the controversy whether, in the general population, refraction increases or decreases during the first years of life. We had noticed that papers reporting a decrease in hypermetropia in early childhood $[1,9,10]$ studied large cross-sections of the general population, whereas papers that reported an initial increase in hypermetropia [4-8] originated from ophthalmological practices or strabismus departments. These conflicting results could be reconciled by assuming a population bias: The children that are referred to an ophthalmologist often have esotropia and maybe esotropia is more likely to occur when hypermetropia increases. Among infants there will be infants with increasing and infants with decreasing hypertropia, with a more or less random distribution. The children who show an increase in hypermetropia after the age that binocular vision develops ( \pm 4 months) and the relation between vergence and accommodation becomes fixed, will need additional accommodation to overcome hypermetropia and, consequently, squint. Two recent studies have corroborated this argument: Aurell \& Norrsell [11] found, in a longitudinal study of children with a family history of strabismus, no strabismus in children whose eyes had emmetropized, in contrast to children without emmetropization who often developed strabismus. Abrahamsson et al. [12, note that the full text of the poster is not in the abstract] found $+1.4 \mathrm{D}$ at age one, on average. At age four, normal children had 0.3D less, but esotropes had 1.29D more hypermetropia. Increasing hypermetropia was risk factor 15.0 for the development of amblyopia, whereas a hypermetropia $\geqslant 3.5 \mathrm{D}$ at age one was only risk factor 4.7 . In this very large study, more than half of all children born in Vasterås in 1979 and 1980 were examined at age one and at age four; 290 of these came to the local eye clinic because of some ophthalmic complaint before age eight. This procedure enabled the authors to relate the occurrence of amblyopia directly to known refractions at ages one and four.

It should be noted that neither study quoted emphasizes the causal relation between increasing hypermetropia and the occurrence of esotropia, but we think their results speak clearly in favour of this. Considering these results, a good procedure for the screening and detection of children with increasing hypertropia seems needed. In any case, in our early refractive screening study we later determined refraction twice, with a six-month interval, in order to be able to estimate the need for glasses more precisely, and prescribe glasses to all children with increasing hypermetropia. 


\section{Acknowledgement}

This study was supported by a grant from the Swiss Union for Prevention of Blindness.

\section{References}

1. Atkinson J, Braddick O, Wattam-Bell J, Durden K, Bobier W, Pointer J. Photorefractive screening of infants and effects of refractive correction. Invest Ophthalmol 1987; 28 (ARVO suppl): 399.

2. Tommila V, Tarkkanen A. Incidence of loss of vision in the healthy eye in amblyopia. Br J Ophthalmol 1981; 65: 575-77.

3. Wang Yu-dong Br J Ophthalmol 1990; 74: 650-53.

4. Brown EVL. Net average yearly changes in refraction of atropinized eyes from birth to beyond middle life. Arch Ophthalmol 1938; 19: 719.

5. Brown EVL. Use-abuse theory of changes in refraction versus biologic theory. Arch Ophthalmol 1942; 28: 845-50.

6. Slapater FJ. Age norms of refraction and vision. Arch Ophthalmol 1950; 43: 466-81.

7. Lahav-Guss C, Kaufmann H. Refraktionsänderungen im Kindesalter: Hyperopie. Klin Mbl Augenheilk 1974; 164: 274-78.

8. Lepard CW. Comparative changes in the error of refraction between fixing and amblyopic eyes during growth and development. Am J Ophthalmol 1975; 80: 485-90.

9. Herrnheiser J. Die Refraktionsentwicklung des menschlichen Auges. Z Heilk 1892; 13: 342-77.

10. Molnár L. Refraktionsuntersuchungen. Acta Med Acad Sci Hung 1967; 23: 297-308.

11. Aurell E, Norrsell K. A longitudinal study of children with a family history of strabismus: factors determining the incidence of strabismus. Br J Ophthalmol 1990; 74: 589-94.

12. Abrahamsson M, Fabian G, Sjöstrand J. Longitudinal changes in refraction and riskindicators for amblyopia. Invest Ophthalmol 1991; 32 (ARVO suppl): 1238.

Address for correspondence: Dr. H.J. Simonsz, Ortoptics \& Neuro-Ophthalmology, Dept. of Ophthalmology, University Hospital Dijkzigt, Dr Molenwaterplein 40, 3015 GD Rotterdam, The Netherlands 